\title{
Toxicidade do extrato hidroalcoólico das folhas de Cissus sicyoides
}

\author{
Gabriela Tafaela Diasa*, Caliandra Maria Bezerra Luna Limaabf, Andressa Brito Liraac, Josue do \\ Amaral Ramalhoa, Kardilandia Mendes de Oliveiraae, Margareth de Fátima Formiga Melo \\ Dinizacdegh

\begin{abstract}
a Laboratório de Ensaios Toxicológicos do Instituto de Pesquisa em Fármacos e Medicamentos, Universidade Federal da Paraíba, João Pessoa, 58051-970, Brasil. *gabrielatafaeladias@gmail.com

b Departamento de Fisiologia e Patologia, Universidade Federal da Paraíba, João Pessoa, 58051-970, Brasil.

c Programa de Pós-Graduação em Produtos Naturais e Bioativos Sintéticos, Universidade Federal da Paraíba, João Pessoa, 58051970, Brasil.

d Departamento de Ciências Farmacêuticas, Universidade Federal da Paraíba, Paraíba, João Pessoa, 58051-970, Brasil.

e Programa de Pós-Graduação em Desenvolvimento e Inovação Tecnológica, Universidade Federal da Paraíba, João Pessoa, 58051 970, Brasil.

f Programa de Pós-Graduação em Modelos de Decisão e Saúde, Universidade Federal da Paraíba, João Pessoa, 58051-900, Brasil. $g$ Pós Graduação em Desenvolvimento e Inovação Tecnológica em Medicamentos, Universidade Federal do Rio Grande do Norte, Lagoa Nova, 59078-970, Brasil.

g Programa de Pós Graduação em Fármaco e Medicamentos da Universidade de São Paulo - USP, São Paulo, 05508-000, Brasil.
\end{abstract}

Recebido: 13 dezembro 2016 / Aceito: 12 janeiro 2017 / Publicado online: 15 fevereiro 2017

\begin{abstract}
Resumo
Cissus sicyoides L. (cipó-anil, insulina vegetal), pertence à família Vitacea, é muito empregada pela população para o tratamento de diabetes. O objetivo desta pesquisa foi realizar uma triagem fitoquímica do extrato hidroalcóolico das folhas de C. sicyoides para utilizá-lo como fonte primária na realização do ensaio toxicológico com Artemia salina L. Foram realizados testes fitoquímicos do extrato hidroalcoólico das folhas de $C$. sicyoides para pesquisa dos metabólitos secundários e foi avaliado a atividade toxicológica utilizando cistos de A. salina para quantificar a concentração letal média (CL50). O extrato apresentou esteróides, taninos, flavonóides e saponinas, compostos que já haviam sido encontrados em outros estudos dessa espécie. Apresentou alta toxicidade frente à A. salina, reportando Concentração Letal Média (CL50) de $930,7 \mathrm{~g} \mathrm{~mL}^{-1}$, servindo de parâmetro para a realização de estudos posteriores em modelos animais com a pretensão de alcançar mais resultados para um uso seguro da planta da $C$. sicyoides.
\end{abstract}

Palavras-chave: Artemia salina, ecotoxicologia, etnobotânica, plantas medicinais, fitoquímica.

\section{Toxicity of the hydroalcoholic extract of the leaves of Cissus sicyoides}

\begin{abstract}
Cissus sicyoides L. (cipó-anil, vegetal insulin) belongs to the family Vitacea. It is much used by the population for the treatment of diabetes. The objective of this research was to perform a phytochemical screening of the hydroalcoholic extract of leaves of C. sicyoides to be used as primary source in the toxicological test with Artemia salina L. Were be phytochemical tests on the hydroalcoholic extract of the leaves of $C$. sicyoides for research on secondary metabolites and was evaluated the toxicological activity using A. saline cysts to quantify the mean lethal concentration (CL50). The extract presented steroids, tannins, flavonoids and saponins, compounds that had already been found in other studies of this species. It presented high toxicity to $A$. salina, reporting a Mean Lethal Concentration (CL50) of $930.7 \mathrm{~g} \mathrm{~mL}^{-1}$, serving as a parameter for the accomplishment of later studies in animal models with the pretension to reach more results for a safe use of $C$. sicyoides.
\end{abstract}

Keywords: Artemia salina, ecotoxicology, ethnobotany, medicinal plants, phytochemistry.

\section{Introdução}

Desde os primórdios, os recursos naturais, em especial as plantas, têm sido um dos elos entre o homem e a natureza, pois os homens pré-históricos já faziam uso das plantas para amenizar o sofrimento dos males físicos que lhes acometiam (Sharma et al., 2014). O homem primitivo, no meio de tentativas e erros, teve de adquirir conhecimentos e através destes e observar quais as plantas poderiam ser utilizadas como alimento, medicamento e ainda destacar as que fossem venenosas ou perigosas. Com o passar do tempo, o poder curativo das plantas, tornou-se muito importante para ser esquecido e o homem começou a sistematizar o seu uso (Allen, 2016). 
Há um crescente estímulo do emprego da fitoterapia em serviços públicos no Brasil, os quais apresentam programas para difusão de sua prática de produção dos fitoterápicos e do cultivo e recomendações do seu uso no momento em que se é obtido o diagnóstico médico. Todavia, existem plantas medicinais que não possuem estudos suficientes para provar a segurança de ingesta, o que pode causar impactos na saúde do consumidor (Alcantara et al., 2015).

A toxicologia avalia os efeitos prejudiciais das substâncias químicas ao organismo vivo, analisando a resposta negativa das substâncias a nível bioquímico, celular e molecular. Atua com um princípio que se fundamenta em explorar o risco da exposição humana frente a diversos produtos para ser possível estabelecer as condições seguras de exposição a estes agentes (Klaassen e Watkins, 2012).

O gênero Cissus L., é uma vasta família Vitaceae, constituído de 300 a 400 espécies de trepadeiras. C. sicyoides $\mathrm{L}$ (C. sicyoides) apresenta vários nomes populares, tais como: cipó-anil, insulina vegetal, anil trepador, cipó-pucá, tinta-dosgentios, uva-brava, cortina-japonesa. É uma trepadeira da América Tropical, originária da República Dominicana, encontrada em grande parte do Brasil, principalmente, no estado do Maranhão e Região Amazônica (Vasconcelos et al., 2007; Banu et al., 2012; Beserra et al., 2016).

O chá das partes aéreas de $C$. sicyoides é utilizado na medicina popular como hipoglicemiantes, anti-hipertensivo e antitérmico. É ainda usado em enfermidades hepáticas, no tratamento das doenças do coração. Apresenta ação anticonvulsivante, sendo por este motivo utilizado em casos de epilepsia e derrame cerebral. Ainda, possui efeito antialérgico, citostático, antibacteriano e gastroprotetor (Vasconcelos et al., 2007; Banu et al., 2012; Beserra et al., 2016). Beltrame et al. (2002) identificaram a partir das partes aéreas de $C$. sicyoides: quercetina 3 - $\alpha$-rhamnoside, cissosides I, II e III, kaempferol 3- $\alpha$-rhamnoside e cissusin.

Diante disso, o objetivo desta pesquisa foi realizar uma triagem fitoquímica do extrato hidroalcóolico das folhas de C. sicyoides para utilizá-lo como fonte primária na realização do ensaio toxicológico com A. salina a fim de determinar concentração média letal (CL50) da planta em estudo.

\section{Material e Métodos}

As atividades de pesquisa não clínica foram realizadas no Laboratório de Ensaios Toxicológicos (LABETOX) do Instituto de Pesquisa em Fármacos e Medicamentos (IPeFarM) da Universidade Federal da Paraíba.

As amostras das folhas de C. sicyoides foram coletadas do Horto de Plantas Medicinais do Instituto de Pesquisa em Fármacos e Medicamentos (IPeFarM) da Universidade Federal da Paraíba (UFPB). A identificação botânica foi realizada pelo setor de botânica do IPeFarM-UFPB. Uma amostra representativa encontra-se no Herbário Lauro Pires Xavier, desta Universidade: Vasconcelos s/n (JPB)

Os cistos de A. salina foram do Laboratório LABETOX, sendo guardados sob resfriamento $\left(5^{\circ} \mathrm{C}\right)$ até a execução do experimento. Foram utilizadas larvas de A. salina, crustáceo da classe Anostracea, (Brine Shrimp Test), na forma de metanáuplio, utilizando-se a Concentração Letal Média
(CL50) como parâmetro de avaliação da atividade toxicológica (Meyer et al., 1982).

\section{Triagem Fitoquímica}

Triagem fitoquímica consiste em uma análise química para detectar presença ou a ausência de metabólitos secundários utilizando reagentes de coloração ou precipitação.

Os testes para pesquisa dos metabólitos secundários foram realizados segundo método desenvolvido Agra e BarbosaFilho (1990), utilizando-se o extrato hidroalcoólico das folhas de $C$. sicyoides, a fim de revelar os seguintes grupos químicos: saponinas,testes de espuma e hemólise; esteróides, pela reação de Liebermann-Buchard; alcalóides, com as reações de Dragendorff, Mayer, Bertrand e Bouchardat; taninos, pela reação com cloreto férrico e reação de Gelatina e; flavonóides, utilizando fita de magnésio e fluorescência.

\section{Bioensaio com A. salina}

O bioensaio com A. salina (Brine Shrimp Test) foi realizado de acordo com McLaughlin e Rogers (1998), permitindo a execução de uma triagem toxicológica que norteou estudos subsequentes. Cerca de $25 \mathrm{mg}$ de cistos de $A$. salina foram incubados em um recipiente dividido em dois compartimentos por uma parede vasada no centro, contendo água do mar à temperatura de 20 a $30^{\circ} \mathrm{C}$, com um $\mathrm{pH}$ entre 8,0 e 9,0. Em um dos compartimentos havia incidência de uma lâmpada de 40 watts, para onde migravam os metanáuplios, obtidos com a eclosão dos cistos após 48 horas de incubação.

Iniciou-se com uma solução de concentração $40 \mathrm{~g} \mathrm{~mL}^{-1}$, as demais concentrações testadas foram 120, 360, 480, 600, 800 e $1000 \mathrm{~g} \mathrm{~mL}^{-1}$. Como não ocorreram mortes nas concentrações de 40 e $120 \mathrm{~g} \mathrm{~mL}^{-1}$, os ensaios foram realizados em triplicata com as demais doses, buscando-se determinar a relação doseresposta, enquanto um grupo controle foi colocado em contato apenas com a água do mar. Cada tubo com a substância -teste, contendo 10 larvas de A. salina, incluindo o controle, foi preenchido até alcançar o volume final de $5 \mathrm{~mL}$ com a água do mar e foram incubadas, no escuro, por 24 horas. As larvas vivas e mortas foram contadas e o valor da CL50 foi determinado pelo método estatístico de Probitos (Parra et al., 2001) utilizando o programa estatístico Microcal Origin 6.0.

\section{Resultados e Discussão}

A triagem fitoquímica evidenciou a presença de esteróides com uma reação moderadamente positiva (++), taninos com uma reação fortemente positiva (+++) e na fluorescência dos flavonóides uma reação moderadamente positiva (Tabela 1).

A análise do Extrato Hidroalcoólico (EHA) das folhas de C. sicyoides corrobora com os estudos de Beserra et. al. (2016), com a mesma espécie, onde reportaram ações terapêuticas antiinflamatória em edema de orelha de ratos pelo excesso de flavonóides presente em $C$. sicyoides, e reportaram que esse constituinte causa do efeito inibitório na motilidade e secreção do fluido intestinal (ação antidiarreica) junto a presença e ação do tanino.

O gênero Cissus, é constituído por cerca de 350 espécies, onde, pelo menos, doze delas são utilizadas na medicina popular para tratar enfermidades (Banu et al., 2012; Tabela 2). 
Tabela 2. Diferentes espécies do gênero Cissus utilizadas na medicina e relação dos constituintes comuns ao gênero. (Adaptada Banu et al., 2012).

\begin{tabular}{|c|c|c|c|c|c|}
\hline Espécie & Localização & Nome Comum & Propriedade medicinal & Constituintes químicos & Referencia \\
\hline C. Sicyoides & $\begin{array}{l}\text { Brasil } \\
\text { (América do } \\
\text { Sul) }\end{array}$ & Insulina vegetal & $\begin{array}{l}\text { Anti-diabético, diurético, anti- } \\
\text { inflamatório, anticonvulsivo, } \\
\text { Ansiolito }\end{array}$ & Esteróides, taninos, flavonóides & $\begin{array}{l}\text { Assob et al. } \\
\qquad(2011)\end{array}$ \\
\hline C. aralioides $\mathrm{L}$. & Camarões & Kindamina & Antimicrobiana & $\begin{array}{l}\text { Alcalóides; Triterpens; Esteróis; Taninos; } \\
\text { Coumarins; Glicosidos; Glicosídeos } \\
\text { cardíacos; Açúcares redutores. }\end{array}$ & $\begin{array}{l}\text { Assob et al. } \\
\qquad(2011)\end{array}$ \\
\hline C. assamica $\mathrm{L}$. & $\begin{array}{l}\text { China, Índia, } \\
\text { Camboja, } \\
\text { Butão, Nepal, } \\
\text { Tailândia }\end{array}$ & - & Veneno anti-serpente & $\begin{array}{l}\text { Lupeol, ácido n-hexacossínico, } \\
\text { isociaridicol-9-O-beta-D-glucopiranósido, } \\
\text { dauco stenina, ácido 3,3'-dimetil elágico, b } \\
\text { sitosterol e bergenina. }\end{array}$ & Xie et al. (2009) \\
\hline C. quadrangulares $\mathrm{L}$. & $\begin{array}{c}\text { Índia, Sri } \\
\text { Lanka (Ásia) }\end{array}$ & Veldt uva & $\begin{array}{l}\text { A cicatrização da fratura, aumenta } \\
\text { a resistência óssea eprotege o osso } \\
\text { da perda óssea pós-menopausa }\end{array}$ & $\begin{array}{l}\text { Flavanoides, esteróis, triterpanóides, } \\
\text { estilbenos, iridoides e } 3,3,4,4 \text { 'tetra } \\
\text { hidroxibifenil }\end{array}$ & Banu et al. (2012) \\
\hline C. ibuensis L. & $\begin{array}{c}\text { Nigéria } \\
\text { (África), Níger, } \\
\text { Gana }\end{array}$ & & $\begin{array}{l}\text { Reumatismo, artrite, Trato } \\
\text { gastrointestinal, Aumento da } \\
\text { proliferação de células de sertoli }\end{array}$ & Quercetina 3-Orutinosida e flavonóides & $\begin{array}{l}\text { Ahmadu et al. } \\
\qquad(2010)\end{array}$ \\
\hline C. rheifolia $\mathrm{L}$. & $\begin{array}{l}\text { Nordeste da } \\
\text { Tailândia }\end{array}$ & $\begin{array}{l}\text { ka-ya-wong } \\
\text { (nome tailandês. } \\
\text { Ttradução:abutre } \\
\text { sem esperança }\end{array}$ & - & $\begin{array}{l}\text { Alcalóides de quinolizidina, flavonóides, } \\
\text { terpenóides }\end{array}$ & $\begin{array}{l}\text { Saifah et al. } \\
\qquad(1983)\end{array}$ \\
\hline C. pallida $\mathrm{L}$. & & & Atividade antifúngica & Estilbenos, triterpenóides e esteróides & Khan et al. (1986) \\
\hline C. verticillata $\mathrm{L}$. & $\begin{array}{l}\text { Trinidade } \\
\text { Tobago } \\
\text { (Caribe) }\end{array}$ & & Anti-colesterol, anti-diabético & $\begin{array}{c}\text { alcalóides, flavonóides, saponinas, taninos } \\
\text { e sais de magnésio, manganês, silício, } \\
\text { cálcio, fósforo e potássio }\end{array}$ & $\begin{array}{l}\text { Silva (1996); } \\
\text { Lans }(2006)\end{array}$ \\
\hline
\end{tabular}


Triagens fitoquímicas foram realizadas utilizando as folhas de $C$. sicyoides por alguns autores que revelou a presença de cumarinas, flavonóides, antocianinas, esteróides e taninos (Xu et al., 2009; Beserra et al., 2016).

Tabela 1. Determinação da triagem fitoquímica do Extrato Hidroalcoólico (EHA) de Cissus sicyoides

\begin{tabular}{|c|c|c|c|}
\hline \multicolumn{3}{|c|}{ Fitoquímica } & \multirow{2}{*}{ Reação } \\
\hline Grupo Químico & & Método & \\
\hline \multirow{3}{*}{ Alcalóides } & & urchardat & - \\
\hline & & Mayer & - \\
\hline & & agendoff & - \\
\hline \multirow{5}{*}{ Esteróides } & Ácidos : & ílico-tungstíco & - \\
\hline & & 0,12 & + \\
\hline & & 0,25 & + \\
\hline & & 0,5 & ++ \\
\hline & Gelatina & 0,5 & + \\
\hline \multirow{3}{*}{ Taninos } & & 1,0 & + \\
\hline & & 2,0 & ++ \\
\hline & $\mathrm{FeCl} 3$ & 0,5 & ++ \\
\hline \multirow{5}{*}{$\begin{array}{c}\text { Flavonóides } \\
\text { Saponinas }\end{array}$} & & 1,0 & ++ \\
\hline & & 2,0 & +++ \\
\hline & Fita $\mathrm{c}$ & e magnésio & + \\
\hline & Flu & prescência & +++ \\
\hline & & Espuma & + \\
\hline
\end{tabular}

(-) Reação negativa, (++) Reação moderadamente positiva, (+) Reação fracamente positiva, (+++) Reação fortemente positiva
O valor médio da CL50 determinado no teste para a espécie C. sicyoides, frente à A. salina, foi de $930,7 \mathrm{~g} \mathrm{~mL}^{-1}$, isto é, essa concentração provoca morte de metade dos indivíduos de uma população.

A. salina é um microcrustáceo amplamente utilizado como indicador de toxicidade em um bioensaio que utiliza a CL50 (Concentração letal 50\%) como parâmetro de avaliação da atividade biológica. A alta toxicidade de um produto frente $A$. salina tenha correlação com ensaio de toxicidade in vivo. Este teste pode ser considerado um bom teste para prever toxicidade oral de produtos, assim como, pode indicar provável potencial para atividade biológica, como antimicrobiana e anticancerígena (Rosa et al, 2016).

Assim, o método de toxicidade com $A$. salina $L$ é um bioensaio utilizado para verificar o potencial dos compostos (Saleh, Z.et al., 2015). Meyer et al. (1982) afirma que, valores de CL50 abaixo de $1000 \mathrm{~g} \mathrm{~mL}^{-1}$, há uma chance do composto ou extrato apresentar potencial bioativo. Sugerindo que $C$. sicyoides possui a presença de potentes componentes bioativos. Ainda, esse resultado corrobora com outras espécies do mesmo gênero, como podemos verificar na Tabela 3.

Desta forma, a espécie em estudo é considerada de alta toxicidade, pois, apresentam CL50 menor que $1000 \mathrm{~g} \mathrm{~mL}^{-1}$. Segundo Meyer et al. (1982) os extratos brutos são classificados em tóxicos (CL50 <1000) e não tóxicos (CL50> 1000; Arcanjo et al,2012; Rosa et al, 2016).

Tabela 3. Toxicidade de algumas plantas medicinais reportadas na literatura.

\begin{tabular}{|c|c|c|c|}
\hline Espécie e Família & Parte & CL50 & Referência \\
\hline $\begin{array}{c}\text { Cissus multistriata } \mathrm{L} . \\
\text { Vitaceae }\end{array}$ & Caule e Folha & $\begin{array}{l}666,98 \mu \mathrm{g} \mathrm{mL}^{-1} \\
512,53 \mu \mathrm{g} \mathrm{mL}^{-1}\end{array}$ & Omale e Okafor (2008) \\
\hline $\begin{array}{l}\text { Cissus adnata } \mathrm{L} . \\
\text { Vitaceae }\end{array}$ & Planta inteira & $1,02 \mu \mathrm{g} \mathrm{mL}^{-1}$ & Rashid et al. (2016) \\
\hline Rafflesiaceae & Capim & $283,21 \mu \mathrm{g} \mathrm{mL}^{-1}$ & Saleh, Z.et al. (2015) \\
\hline $\begin{array}{c}\text { Tropaeolum papillosum } \mathrm{L} . \\
\text { Tropaeolaceae }\end{array}$ & Raiz & $719,95 \mu \mathrm{g} / \mathrm{mL}^{-1}$ & Saleh, Z.et al. (2015) \\
\hline $\begin{array}{l}\text { Costus Pisonis L. } \\
\text { Costaceae }\end{array}$ & Partes aéreas & $389,26 \mathrm{ppm}$ & Brasileiro, et al. (2006) \\
\hline $\begin{array}{l}\text { Cymbopogon nardus } \mathrm{L} . \\
\text { Poaceae }\end{array}$ & Folhas & $118,92 \mathrm{ppm}$ & Brasileiro, et al. (2006) \\
\hline $\begin{array}{c}\text { Eleutherine bulbosa (Mill.) (bulb) } \\
\text { Iridaceae }\end{array}$ & Folhas & $269,59 \mathrm{ppm}$ & Brasileiro, et al. (2006) \\
\hline $\begin{array}{c}\text { Erygium foetidium } \mathrm{L} . \\
\text { Apiaceae }\end{array}$ & Partes aéreas & $885,37 \mathrm{ppm}$ & Brasileiro, et al. (2006) \\
\hline
\end{tabular}

O uso de plantas é uma prática prevalente na população, principalmente onde as condições sociais e econômicas desfavoráveis (Balbinot et al., 2013; Messias et al., 2015). A C. sicyoides é amplamente usada na medicina popular brasileira, uma vez que, a presença de metabólitos secundários como agente diurético, anti-inflamatório, antidiabético, anti-gripal, dentre outras ações que comprova efetividade em relatados científicos e na vivência popular (Silva et al., 2012).

\section{Conclusões}

A triagem fitoquímica do extrato hidroalcoólico $C$. sicyoides evidenciou a presença dos constituintes químicos característico do gênero Cissus que são responsáveis pela ação biológica da planta. Ainda, foi evidenciada a presença de potentes componentes bioativos frente ao modelo experimental com A. salina. Assim, é relevante pesquisas posteriores com estudos toxicológicos subsequentes experimentos em animais, buscando obter melhores resultados sobre alterações hematológicas, bioquímicas, comportamentais, letalidade, histopatológicas e mutagênicas com a pretensão de alcançar uso seguro da $C$. sicyoides.

\section{Referências}

Agra, M.F.; Barbosa-Filho, J.M. 1990. Levantamento da flora medicinal da Paraíba e triagem fitoquímica. Revista Brasileira de Farmácia, 71(3): 7276. 
Ahmadu, A.A.; Onanuga, A.; Aquino, R. 2010. Flavonoid glycosides from the leaves of Cissus ibuensis hook (vitaceae). African Journal of Traditional, Complementary and Alternative Medicines, 7(3): 225-230.

Alcantara, R.G.L.; Joaquim, R.H.V.T.; Sampaio, S.F. 2016. Plantas medicinais: o conhecimento e uso popular. Revista de APS, 18(4): 470482.

Allen Jr, L.V. 2015. Introdução à Farmácia de Remington. Artmed Editora, Porto Alegre, p. 9-26.

Arcanjo, D.D.R.; Albuquerque, A.C.M.; Melo-Neto, B.; Santana, L.C.L.R.; Medeiros, M.; Citó, A.M.G.L. 2012. Bioactivity evaluation against Artemia salina Leach of medicinal plants used in Brazilian Northeastern folk medicine. Brazilian Journal of Biology, 72(3): 505509.

Balbinot, S.; Velasquez, P.G.; Düsman, E. 2013. Reconhecimento e uso de plantas medicinais pelos idosos do Município de MarmeleiroParaná. Revista Brasileira de Plantas Medicinais, 15(4), 632-638.

Banu, J. 2012. Medicinal properties of plants from the genus Cissus: A review. Journal of Medicinal Plants Research, 6(16): 3080-3086.

Beltrame, F.; Ferreira, A.; Cortez, D. 2002. Coumarin glycoside from Cissus sicyoides. Natural product letters, 16(4): 213-216.

Beserra, F.P.; Santos, R.C.; Périco, L.L.; Rodrigues, V.P.; Kiguti, L. R.A.; Saldanha, L.L.; ... Hiruma-Lima, C. A. 2016. Cissus sicyoides: Pharmacological Mechanisms Involved in the Anti-Inflammatory and Antidiarrheal Activities. International journal of molecular sciences, 17(2): 149.

Brasileiro, B.G.; Pizziolo, V.R.; Raslan, D.S.; Jamal, C.M.; Silveira, D. 2006. Antimicrobial and cytotoxic activities screening of some Brazilian medicinal plants used in Governador Valadares district. Revista Brasileira de Ciências Farmacêuticas, 42(2): 195-202.

Khan, M.A.; Nabi, S.G.; Prakash, S.; Zaman, A. 1986. Pallidol, a resveratrol dimer from Cissus pallida. Phytochemistry, 25(8): 1945-1948.

Klaassen, C.D.; Watkins III, J.B. Fundamentos em toxicologia de Casarett e Doul. 2. ed. Porto Alegre: AMGH, 2012. 472p.

Lans, C.A. 2006. Ethnomedicines used in Trinidad and Tobago for urinary problems and diabetes mellitus. Journal of ethnobiology and ethnomedicine, 2(1): 45.

Mclaughlin, J.L.; Rogers, L.L.; Anderson, J.E. 1998. The use of biological assays to evaluate botanicals. Drug information journal, 32(2): 513 524.

Meyer, B.N.; Ferrigni, N.R.; Putnam, J.E.; Jacobsen, L.B.; Nichols, D.J.; McLaughlin, J.L. 1982. Brine shrimp: a convenient general bioassay for active plant constituents. Planta medica, 45(05): 31-34.

Messias, M.C.T.B.; Menegatto, M.F.; Prado, A.C.C.; Santos, B.R.; Guimarães, M.F.M. 2015. Popular use of medicinal plants and the socioeconomic profile of the users: a study in the urban area of Ouro Preto, Minas Gerais, Brazil. Revista Brasileira de Plantas Medicinais, 17(1): 76-104.

Omale, J.; Okafor, P.N. 2008. Comparative antioxidant capacity, membrane stabilization, polyphenol composition and cytotoxicity of the leaf and stem of Cissus multistriata. African Journal of Biotechnology, 7(17): 3129-3133.

Parra, A.L.; Yhebra, R.S.; Sardiñas, I.G.; Buela, L.I. 2001. Comparative study of the assay of Artemia salina L. and the estimate of the medium lethal dose (LD50 value) in mice, to determine oral acute toxicity of plant extracts. Phytomedicine, 8(5): 395-400.

Rashid, R.B.; Towsif, F.N.; Bushra, F.A.; Tahia, F. 2016. Antioxidant Membrane Stabilizing and Cytotoxic Activities of Cissus adnata (Roxb.). Dhaka University Journal of Pharmaceutical Sciences, 15(1): 69-71.

Rosa, C.; Veras, K.; Silva, P.; Lopes Neto, J. J.; Cardoso, H.; Alves, L.; ... MORAES, D. 2016. Composição química e toxicidade frente Aedes aegypti L. e Artemia salina Leach do óleo essencial das folhas de Myrcia sylvatica (G. Mey.) DC. Revista Brasileira de Plantas Medicinais, 18(1): 19-26.

Saifah, E.; Kelley, C.J.; Leary, J.D. 1983. Constituents of the leaves of Cissus rheifolia. Journal of natural products, 46(3): 353-358.

Saleh, Z.; Zuhud, E.A.M.; Sari, R.K. 2015. Phytochemical screening and antioxidant activity of ethanolic extract of Rhizanthes deceptor (Rafflesiaceae) and its host Tetrastigma papillosum. Research Journal of Medicinal Plant, 9(6): 293-299.

Sharma, C.; Rajendar, K.; Kumari, T.; Arya, K.R. 2014. Indian traditional therapies and bio-prospecting: their role in drug development research. International Journal of Pharmaceutical Sciences and Research, 5(3): 730-741.
Silva, G.A.D.; Araújo, L.C.; Oga, S.; Akisue, G. 1996. Estudo toxicológico e farmacológico dos extratos fluídos de Cissus sicyoides L. Revista Brasileira de Farmacognosia, 5(2): 143-155.

Silva, M.I.; Melo, C.T.; Vasconcelos, L.F.; Carvalho, A.M.; Sousa, F.C. 2012. Bioactivity and potential therapeutic benefits of some medicinal plants from the Caatinga (semi-arid) vegetation of Northeast Brazil: a review of the literature. Revista Brasileira de Farmacognosia, 22(1): 193207.

Vasconcelos, T.H.C.D.; Modesto-Filho, J.; Diniz, M.F.F.M.; Santos, H.B.; Aguiar, F.B.D.; Moreira, P.V.L. 2007. Estudo toxicológico pré-clínico agudo com o extrato hidroalcoólico das folhas de Cissus sicyoides L. (Vitaceae). Revista Brasileira de Farmacognosia, 17(4): 583-591.

Xie, Y.H.; Deng, P.; Zhang, Y.Q.; Yu, W.S. 2009. Studies on the chemical constituents from Cissus assamica. Journal of Chinese medicinal materials, 32(2): 210-213.

Xu, F.; Matsuda, H.; Hata, H.; Sugawara, K.; Nakamura, S.; Yoshikawa, M. 2009. Structures of new flavonoids and benzofuran-type stilbene and degranulation inhibitors of rat basophilic leukemia cells from the Brazilian herbal medicine Cissus sicyoides. Chemical and Pharmaceutical Bulletin, 57(10): 1089-1095. 